\title{
Instrumentos de medida de adherencia al tratamiento
}

\author{
X. NOGUÉS SOLÁN, M. L. SORLI REDÓ, J. VILLAR GARCÍA \\ Unitat de Recerca en Fisiopatología Óssia i Articular (URFOA). Servicio de Medicina \\ Interna. Hospital del Mar. Institut Municipal d'Investigació Médica (IMIM). Universitat \\ Autónoma de Barcelona. Hospital del Mar. Barcelona
}

TOOLS TO MEASURE TREATMENT ADHERENCE

\begin{abstract}
RESUMEN
La medición de la adherencia a los tratamientos es necesaria, sin embargo aunque las herramientas con este propósito son diversas, la mayoría de ellas no cumplen su cometido a la perfección. La adherencia engloba dos conceptos: cumplimiento de dosis y forma de administración y persistencia en la duración del tratamiento prescrito. Uno de los instrumentos de medición de la adherencia son las bases de datos de farmacia. Se ha demostrado utilizando esta herramienta de medición que la persistencia de las pacientes que reciben tratamiento para la osteoporosis puede llegar a ser menor del $25 \%$ tras un año de la prescripción. Otro instrumento muy utilizado es el recuento de los comprimidos no tomados, que dará el porcentaje de cumplimiento en un periodo establecido. Un cuestionario autoadministrado muy útil es el cuestionario de Morisky-Green validado en su versión española por Val Jiménez y cols. Este cuestionario consiste en cuatro sencillas y cortas preguntas. Recientemente se han introducido los métodos electrónicos que permiten conocer cuándo y a qué hora el paciente adquiere la medicación. La medición de la adherencia no es fácil y es necesario utilizar varios métodos a la vez para evitar la sobreestimación. En enfermedades que precisan un tratamiento a largo plazo como la osteoporosis se ha de aumentar la adherencia para prevenir fracturas.
\end{abstract}

PALABRAS CLAVE: Adherencia. Herramientas de medición. Osteoporosis. Tratamiento.

\begin{abstract}
Adherence measurement of treatments is necessary, in spite there are several tools the most of them do not achieve fully the goal. Adherence is a word that includes two concepts: compliance to doses and administration way and persistence on the treatment time. One of these tools is the pharmacy data base. It has been demonstrated using this tool that persistence for osteoporosis patient treatments could be fewer than $25 \%$ after one year of prescription. An extended tool is the count of not taked pills that inform of adherence percentage in a period of time. One very useful self- administrated questionnaire is the Morisky-Green with a Spanish version validated by Val Jimenez et al. This questionnaire consists of four simple and short questions. Recently it has been introduced electronic methods that permit to know when and what time the patient takes the pill. Measurement of adherence is not easy and it is necessary to use some tools at the same time to avoid overestimation. Diseases with a long period of treatment as osteoporosis the increase of adherence is needed to prevent fractures.
\end{abstract}

KEY WORDS: Adherence. Measure tools. Osteoporosis. Treatment.

Nogués Solán X, Sorli Redó ML, Villar García J. Instrumentos de medida de adherencia al tratamiento. An Med Interna (Madrid) 2007; 24: 138-141.

\section{INTRODUCCIÓN}

El avance en el tratamiento de las enfermedades se ha basado en la formación continuada de los facultativos, la mejora de las técnicas de diagnóstico y el desarrollo de nuevas terapias. Los ensayos clínicos controlados representan los instrumentos que prueban la eficiencia de un fármaco en una cohorte seleccionada, controlada y monitorizada de forma estricta. Sin embargo, el paso del fármaco a la población general hace que se pierda efectividad debido a la falta de cumplimiento por el paciente, tanto en lo que se refiere a la dosificación como a la duración (1).

Medir la adherencia al tratamiento es necesario si se quiere optimizar el manejo terapéutico de las enfermedades. Las herramientas con este propósito son diversas, sin embargo, la mayoría de ellas no cumplen su cometido a la perfección.

La adherencia engloba dos conceptos: cumplimiento de dosis y forma de administración (compliance para los anglo-

Trabajo aceptado: 19 de octubre de 2006 
sajones) y persistencia en la duración del tratamiento prescrito. Según ambos conceptos, se pueden identificar diversos métodos de medición. Teóricamente el máximo cumplimiento terapéutico, tanto de dosis prescrita como de forma de administración, estaría reflejado en la relación (comprimidos tomados/comprimidos prescritos) y sería del $100 \%$ si en la fórmula numerador y denominador fueran iguales.

La fórmula sería sencilla de cumplir, si realmente el paciente respondiera al facultativo correctamente a la simple cuestión de ¿cuántos comprimidos se ha tomado desde la ultima visita? Sin embargo el problema surge cuando o bien el paciente no se acuerda o bien con un sentimiento de culpa por haberse olvidado algún comprimido falsea el resultado, unas veces por vergüenza y otras por no desengañar al médico.

Se han de buscar pues fórmulas fáciles para evitar ese interrogatorio directo que puede incomodar tanto al paciente como al facultativo.

\section{BASES DE DATOS DE FARMACIA}

Un instrumento que da una buena aproximación al problema es la base de datos de farmacia. Este es un instrumento útil, sobre todo para la industria farmacéutica y la administración, pues permite conocer el número de nuevos tratamientos y la discontinuación de los mismos. En nuestro país se consigue una buena aproximación a los datos reales pues las prescripciones de fármacos por la mayoría de facultativos están incluidas dentro del Sistema Nacional de Salud, que subvenciona la totalidad del fármaco o bien del $60 \%$ de su precio. Además las prescripciones son nominales y desde la introducción de la tarjeta sanitaria y la informatización del sistema es posible conocer los datos de inicio y final de tratamiento para un paciente determinado. El lado negativo de este sistema viene dado porque, a pesar de realizarse la prescripción y de que la farmacia administre el fármaco, no se tiene la seguridad de que el paciente cumpla realmente el tratamiento en la dosis y la forma adecuada. Sí permite, sin embargo, conocer el gasto sanitario de un fármaco determinado y, por lo tanto, hacer previsiones de mercado para el futuro.

Algunos autores han utilizado esta metodología para analizar la persistencia a un tratamiento determinado en grandes bases de datos poblacionales. Así Faulkner y cols. (2) analizaron de forma retrospectiva el seguimiento en la prescripción de tratamiento hormonal sustitutivo en una cohorte de 28.718 mujeres posmenopáusicas, de edades comprendidas entre los 40 y 60 años. Concluyeron que después de un año un $54,4 \%$ de las mujeres se podía considerar como no cumplidora pues la relación entre fármaco prescrito y fármaco retirado de la farmacia no llegaba al $75 \%$. También Mc Combs y cols. (3) utilizaron una extensa base de datos de una compañía aseguradora con más de 51.000 pacientes, para estudiar la adherencia a los diversos tratamientos para la osteoporosis, terapia hormonal, raloxifeno y bifosfonatos. Aunque en este estudio la mayoría de las pacientes recibía terapia hormonal, en los tres grupos de tratamiento se observó una persistencia de menos de un $25 \%$ de las pacientes tras el año de la prescripción.

Esta metodología da un conocimiento global sobre la adherencia a una medicación pero evidentemente no permite la extensión a un caso particular. Tampoco es posible, debido a las leyes de protección y confidencialidad de datos, solicitar información de un paciente determinado para conocer si retira de la farmacia las prescripciones de los facultativos.

Así pues las bases de datos de farmacia o compañías aseguradoras con reembolso de precio permiten una aproximación al cumplimiento de forma poblacional y para grandes cohortes, pero no permiten extrapolar datos a cohortes pequeñas o pacientes individualizados.

INFORMACIÓN DEL PROPIO PACIENTE DE SU ADHERENCIA O COMPORTAMIENTO

El paciente puede proporcionar directamente los datos de su comportamiento en cuanto la adherencia mediante diversas fórmulas. Una muy utilizada en los ensayos clínicos controlados es la del retorno de los envases o blísters. El recuento de los comprimidos no utilizados dará el porcentaje de cumplimiento en un periodo establecido. Éste es un procedimiento que no puede asegurar si el paciente se ha tomado la medicación de forma correcta y a la hora prescrita. Además tampoco se puede asegurar que el paciente retorne todos los envases con comprimidos no utilizados, por lo que entre los pacientes con bajo cumplimiento el recuento de comprimidos es sospechosamente erróneo (4).

Otro sistema muy utilizado es la información proporcionada por el propio paciente o patient-self-report (5) que combina dos tipos de preguntas referidas a la dificultad y a los comprimidos olvidados en el último mes (Tabla I).

El cumplimiento se valora mediante la fórmula: 30 - comprimidos olvidados $x 100$.

También se puede utilizar el cuestionario de cumplimiento preguntando: ¿está el paciente siguiendo el tratamiento?, y si no lo hace, ¿cuál ha sido la razón para el abandono?

Finalmente el cuestionario de forma autorreferida más utilizado es el de Morisky-Green (6) validado en su versión española por Val Jiménez y cols. (7) (Tabla II). Este cues-

\section{TABLA I}

CUESTIONARIO DE AUTOCUMPLIMIENTO

- ¿Tiene alguna dificultad para tomar las pastillas? (Do you have difficulty in taking the tablets?)

- ¿Cuántas pastillas ha olvidado en el último mes? (How many tablets have you missed in the last month?)

\section{TABLA II}

CUESTIONARIO DE MORISKY-GREEN (TRADUCIDO)

- ¿Se olvida alguna vez de tomar el medicamento?

- ¿Toma la medicación a la hora indicada?

- Cuando se encuentra bien, ¿deja alguna vez de tomar la medicación?

- Si alguna vez se sienta mal, ¿deja de tomar la medicación? 
tionario se ha utilizado en numerosos estudios de adherencia de fármacos antihipertensivos $(8,9)$ y antirretrovirales para SIDA (10,11). En osteoporosis también algunos estudios lo han utilizado para demostrar la efectividad de diversos métodos para incrementar la adherencia al tratamiento $(12,13)$.

\section{MÉTODOS ELECTRÓNICOS}

Los instrumentos de medida de adherencia administrados para que el propio paciente responda tienen la tendencia a sobreestimar el cumplimiento. Éste es un dato conocido, así cuando se combinan con métodos como el retorno de los envases existe un cierto desajuste (4). Por este motivo se han desarrollado diversos dispositivos electrónicos como Electronic monitoring device (medication event monitoring system MEMS) que registra el momento en que el paciente abre el frasco de la medicación (14) o un dispensador electrónico de medicación desarrollado específicamente para mejorar la adherencia de la población geriátrica (15).

La incorporación de métodos electrónicos permite conocer más datos sobre cuándo y a qué hora el paciente adquiere la medicación, sin embargo no dice si el paciente ingiere correctamente la medicación. Los datos derivados de los estudios en los que se han utilizado estos instrumentos de medida electrónicos demuestran que tan sólo un 50-60\% de pacientes consigue una adherencia óptima o excelente (16).

\section{MONITORIZACIÓN MEDIANTE PARÁMETROS ANALÍTICOS}

Evidentemente la determinación sanguínea de los niveles plasmáticos de un fármaco sería la forma ideal de monitori- zar la adherencia de un paciente a la medicación prescrita. Sin embargo, es obvio que ello es imposible excepto dentro de ensayos clínicos específicos. No obstante pueden existir parámetros analíticos de respuesta al fármaco que permitan la monitorización de la respuesta al tratamiento versus la enfermedad. Ejemplos de ello son los tratamientos con acenocumarol y el tiempo de protrombina o la L-tiroxina y la TSH. En osteoporosis los marcadores bioquímicos de remodelado pueden ser utilizados como respuesta al tratamiento. Clowes y cols. (17) han demostrado una buena correlación entre la buena adherencia y la respuesta medida mediante $\mathrm{N}$ telopéptido urinario al tratamiento con antirresortivos con una correlación $r=-0,36$.

Se ha de considerar sin embargo que los marcadores de remodelado presentan una variabilidad entre el 7 y el $16 \%$ $(18,19)$, pero algunos autores ya recomiendan su uso como instrumento de control de la adherencia y para localizar a los pacientes con bajo cumplimiento (20).

\section{CONCLUSIÓN}

Una adherencia baja a los fármacos produce disminución de la efectividad del fármaco en la población general. La medición de la adherencia no es fácil y en muchas ocasiones se precisa de varios métodos para evitar la sobreestimación. Un método muy utilizado es el cuestionario de Morisky-Green que, combinado con el recuento de comprimidos, permite una buena aproximación al resultado.

En enfermedades que precisan un tratamiento a largo plazo como la osteoporosis se ha de aumentar el grado de adherencia para conseguir disminuir los eventos finales como son las fracturas. La administración de pautas de tratamiento más espaciadas y simples para los pacientes como formulaciones semanales o mensuales pueden mejorar la adherencia respecto a las dosis diarias (21).

\section{Bibliografía}

1. Adherence to long-term-therapies: evidence for action. WHO report 2003.

2. Faulkner DL, Young C, Hutchins D, McCollam JS. Patient noncompliance with hormone replacement therapy: A nationwide estimate using a large prescription claims database. Menopause 1998; 5: 2269.

3. McCombs JS, Thiebaud P, McLaughlin-Miley C, Shi J. Compliance with drug therapies for the treatment and prevention of osteoporosis. Maturitas 2004; 48: 271-87.

4. Rudd P, Byyny RL, Zachary V, LoVerde ME, Titus C, Mitchell $\mathrm{WD}$, et al. The natural history of medication compliance in a drug trial: Limitations of pill counts. Clin Pharmacol Ther 1989; 46: 16976.

5. Haynes RB, Sackett DL, Gibson ES et al. Improvement of medication compliance in uncontrolled hypertension. Lancet 1976; 1: 1265-8.

6. Morisky DE, Green LW, Levine DM. Concurrent and predictive validity of a self-reported measure of medication adherence. Med Care 1986; 24: 67-74.

7. Val Jiménez A, Amorós G, Martínez P, Ferández ML, León M. Estudio descriptivo del cumplimiento del tratamiento farmacológico anti- hipertensivo y validación del test Morisky y Green. Aten Primaria 1992; 10: 767-70.

8. Pineiro F, Gil V, Donis M, Orozco D, Pastor R, Merino J. Validez de 6 métodos indirectos para valorar el cumplimiento del tratamiento farmacológico en la hipertensión arterial. Aten Primaria 1997; 19: 372-5.

9. Shalansky SJ, Levy AR, Ignaszewski AP. Self-reported Morisky score for identifying nonadherence with cardiovascular medications. Ann Pharmacother 2004; 38: 1363-8.

10. Corless IB, Kirksey KM, Kemppainen J, Nicholas PK, McGibbon C, Davis SM, et al. Lipodystrophy-associated symptoms and medication adherence in HIV/AIDS. AIDS Patient Care STDS 2005; 19: $577-86$.

11. Pratt RJ, Robinson N, Loveday HP, Pellowe CM, Franks PJ, Hankins $\mathrm{M}$, et al. Adherence to antiretroviral therapy: appropriate use of selfreporting in clinical practice. HIV Clin Trials 2001; 2: 146-59.

12. Turbi C, Herrero-Beaumont G, Acebes JC, Torrijos A, Grana J, Miguélez R, Sacristán J, Marín F. Compliance and satisfaction with raloxifene versus alendronate for the treatment of postmenopausal osteoporosis in clinical practice: An open-label, prospective, nonrandomized, observational study. Clin Ther 2004; 26: 245-56. 
13. Guilera M, Fuentes M, Grifols M, Ferrer J, Badía X; OPTIMA study investigators. Does an educational leaflet improve self-reported adherence to therapy in osteoporosis? The OPTIMA study. Osteoporos Int 2006; 17: 664-71.

14. Márquez-Contreras E, Martell-Clarós N, Gil-Guillén V, De la FigueraVon Wichmann M, Casado-Martínez JJ, Martín-de Pablos JL, et al. Compliance Group of the Spanish Society of Hypertension. Efficacy of a home blood pressure monitoring programme on therapeutic compliance in hypertension: the EAPACUM-HTA study. J Hypertens 2006; 24: 169-75.

15. Buckwalter KC, Wakefield BJ, Hanna B, Lehmann J. New technology for medication adherence: Electronically managed medication dispensing system. J Gerontol Nurs 2004; 30: 5-8.

16. Miller NH. Compliance with treatment regimens in chronic asymptomatic diseases. Am J Med 1997; 102 (2A): 43-9.

17. Clowes JA, Peel NF, Eastell R. The impact of monitoring on adherence and persistence with antiresorptive treatment for postmenopausal osteoporosis: a randomized controlled trial. J Clin Endocrinol Metab 2004 89: 1117-23.

18. Garnero P, Borel O, Delmas PD. Evaluation of a fully automated serum assay for C-terminal cross-linking telopeptide of type I collagen in osteoporosis. Clin Chem 2001; 47: 694-702.

19. Eastell R, Mallinak N, Weiss S, Ettinger M, Pettinger M, Cain D, et al. $3^{\text {rd }}$. Biological variability of serum and urinary N-telopeptides of type I collagen in postmenopausal women. J Bone Miner Res 2000; 15: 594-8.

20. Roux C, Garnero P, Thomas T, Sabatier JP, Orcel P, Audran M; Comite Scientifique du GRIO. Recommendations for monitoring antiresorptive therapies in postmenopausal osteoporosis. Joint Bone Spine 2005; 72: 26-31.

21. Reginster JY, Rabenda V. Adherence to treatment in osteoporosis influence on efficiency. Rev Med Suisse 2005; 1: 2278-81. 